\title{
RESEARCH HIGHLIGHT OPEN Gasdermin: a novel therapeutic target for tumour treatment by activating anti-tumour immunity
}

\author{
Caiyun $\mathrm{Fu}^{1}$ \\ Signal Transduction and Targeted Therapy (2020)5:69
}

; https://doi.org/10.1038/s41392-020-0180-4

\begin{abstract}
A very recent study by Zhang et al. published in Nature demonstrates that gasdermin E (GSDME, also known as DFNA5) is a tumour suppressor by activating caspaseindependent pyroptosis to enhance anti-tumour immunity. In the meantime, a study by Wang et al. published in Nature also demonstrates the anti-tumour effect caused by gasdermin A3 (GSDMA3) to induce pyroptosis requires both cytotoxic $\mathbf{T}$ cells and $\mathrm{CD}^{+} \mathrm{T}$ helper cells. ${ }^{2}$ The authors intriguingly showed that pyroptosis-induced inflammation triggers robust anti-tumour immunity, thus gasdermin is a promising novel therapeutic target for tumour treatment (Fig. 1).

Pyroptosis is one kind of programmed necrosis mediated by the gasdermin family. ${ }^{3}$ The gasdermin family includes GSDMD, GSDMA, GSDMB, GSDMC, GSDME, and DFNB59. GSDMD is identified as the pyroptotic substrate of caspase-1/4/5/11 and multiple gasdermin- $\mathrm{N}$ domains can bind membrane lipids and bear a membrane poreforming activity with the inner diameters of $10-14 \mathrm{~nm}$ of most gasdermin pores. ${ }^{4}$ The gasdermin pores disrupt the balance of the cell membrane to induce water inflow and cell swelling, as well as the release of many proinflammatory cytokines. ${ }^{5}$ GSDME can be cleaved by caspase 3 to switch apoptosis to pyroptosis. Although the expression level of GSDME is suppressed with the potential as a tumour suppressor, it is still unclear whether and how GSDME acts as a tumour suppressor.
\end{abstract}

The Judy Lieberman group demonstrated that GSDME function was reduced in 20 of 22 tested cancer-associated GSDME mutation. ${ }^{1}$ Zhang et al. addressed the issue of GSDME function in tumour treatment in vitro and in vivo. GSDME function of tumour suppression was mediated by activating caspaseindependent pyroptosis, which depended on an involvement of the enhancement of phagocytosis of tumour cells by tumourassociated macrophages, as well as the number and functions of tumour-infiltrating natural-killer and $\mathrm{CD}^{+}$T lymphocytes. ${ }^{1}$

Using The Cancer Genome Atlas database, the authors first analysed the expression levels of Gsdme messenger RNA and/or protein in primary breast cancers and colorectal cancer to seek the tumour model with GSDME high or poor expression level. Gsdme was knocked out in highly expressing cell lines (EMT6, CT26, and SH-SY5Y), or stably expressed in poorly expressing cell lines (B16F10, 4T1E, and HeLa). The tumours knocked out for Gsdme in highly expressing cells grew much faster accompanied by fewer $\mathrm{CD}^{+} \mathrm{T}$ and NK cells, as well as tumour-associated macrophages. Meanwhile, tumour-infiltrating lymphocytes (TILs: CD8 ${ }^{+} \mathrm{T}$ and NK cells) from $\mathrm{Gsdme}^{-/-}$tumours also expressed less granzyme B $(\mathrm{GzmB})$, perforin (PFN), interferon- $\gamma$ (IFN $\gamma)$, and TFN. The overexpression of Gsdme in cells with poorly expressing of Gsdme had the opposite effect. These results indicated that GSDME has the function of tumour suppression and TIL promotion.

Next, the authors wanted to know whether the tumour suppression of GSDME was mediated by enhanced immune function. The authors provided further evidence that GSDMEmediated tumour inhibition was reduced significantly in mice lacking either $\mathrm{CD}^{+}{ }^{+} \mathrm{T}$ or NK cells, indicating that killer lymphocytes mediate tumour suppression of GSDME. To determine whether killer lymphocytes induced pyroptosis, the authors used human NK line YT or NK-92 incubated with empty-vector and hGSDME-overexpressing HeLa cells. The results showed that NK cells induced pyroptosis in caspase-dependent and caspase-independent manners. Moreover, the authors used ethylene glycoltetraacetic acid (EGTA) to inhibit cytotoxic granule release and PFN with the results that EGTA completely blocked pyroptosis. All these results indicated that pyroptosis activated by killer cells depended on cytotoxic granule release.

Furthermore, the authors speculated that Gzm proteases were involved in NK-induced pyroptosis by cleavage of GSDME. Based on the results of co-incubation GzmB and GSDME or GSDME D270A mutant, the authors determine that GzmB cleaved GSDME at D270, the same site of caspase 3 . Then, the authors hypothesised that mutation of this residue in tumours should abrogate tumour suppression. B16 and 4T1E cells were used to test this hypothesis. The results showed that only tumours overexpressing wild-type GSDME reduced tumour growth, while tumours overexpressing D270A GSDME or empty vector grew with no difference. The authors provided further evidence that the function of killer lymphocytes $\left(\mathrm{CD}^{+} \mathrm{T}\right.$ or NK cells) was enhanced in tumours with the overexpression of wild-type GSDME. All these results provided evidence that cleavage of GSDME at D270 to disturb cell membranes via pore formation was required for tumour suppression.

Altogether, the study by Zhang and colleagues $^{1}$ elegantly illustrates how GSDME acted as a tumour suppressor by inducing pyroptosis in melanoma, triple-negative breast cancer, and colorectal cancer tumours. The enhancement of anti-tumour killer-cell cytotoxicity was necessary and essential for tumour inhibition of GSDME. GSDME cleavage at D270 by GzmB/caspase 3 promoted pore formation to induce pyroptosis and suppress tumour growth through the enhancement of anti-tumour adaptive immunity.

In the meantime, Feng Shao and Zhibo Liu groups ${ }^{2}$ established a bioorthogonal chemical system to enable the controlled release of a drug from an antibody-drug conjugate in mice. Using this bioorthogonal system, the authors observed membrane enrichment of gasdermin $\mathrm{N}$ domains after GSDMA3 conjugated to 60-nm nanoparticles and pyroptotic morphology, which phenomenon was

\footnotetext{
${ }^{1}$ Zhejiang Provincial Key Laboratory of Silkworm Bioreactor and Biomedicine, College of Life Sciences and Medicine, Zhejiang Sci-Tech University, Hangzhou 310018, China Correspondence: Caiyun Fu (fucy03@zstu.edu.cn)
}

Received: 30 April 2020 Accepted: 3 May 2020

Published online: 13 May 2020 
$\mathrm{Fu}$

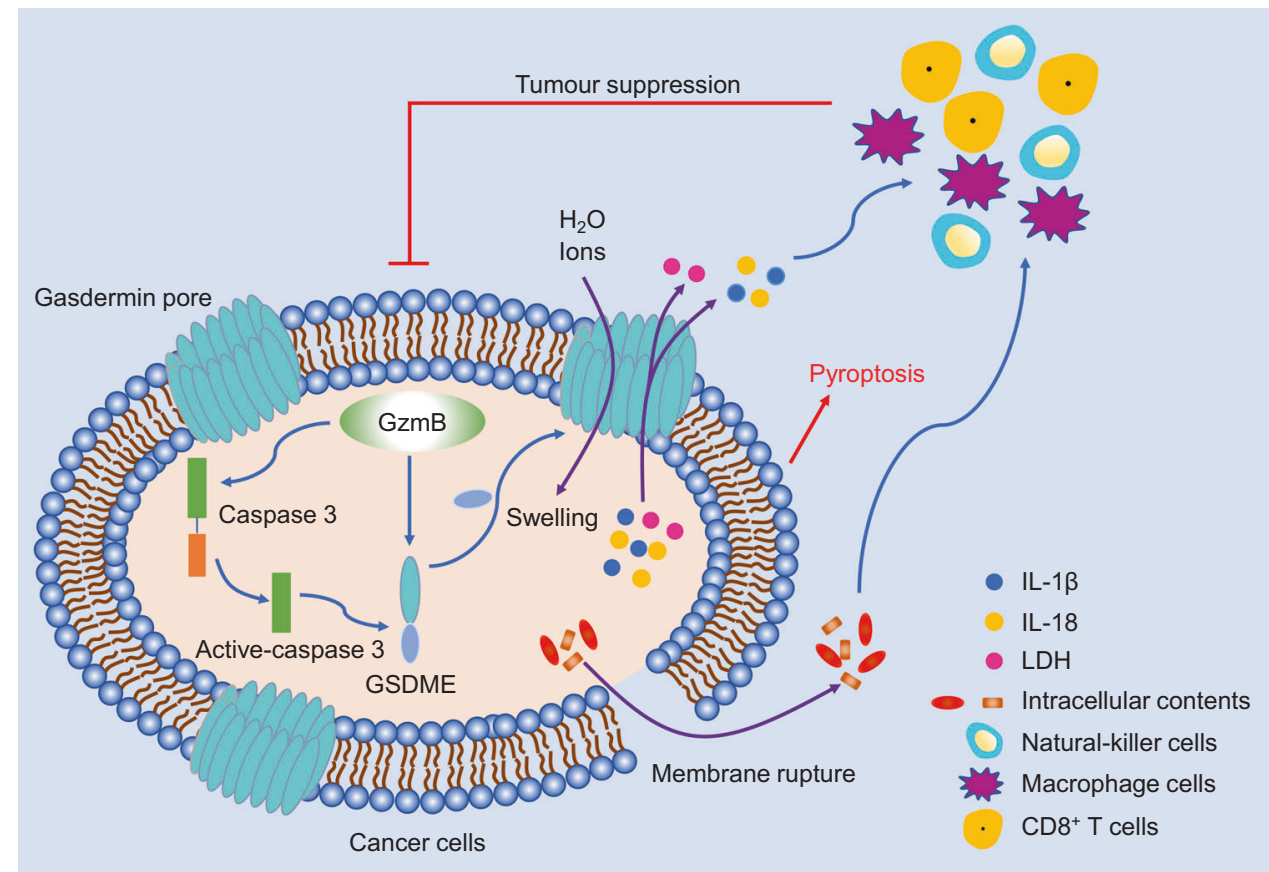

Fig. 1 GSDME cleavage by GzmB/caspase 3 promoted pore formation to induce pyroptosis and suppress tumour growth through the enhancement of anti-tumour adaptive immunity

deficient when GSDMA3 was mutant with E14K and L184D. Furthermore, the tumour regression induced by GSDMA3 conjugated to nanoparticles was inhibited by IL-1 $\beta$ antibody markedly. The authors also demonstrated that treatment with GSDMA3 conjugated to nanoparticles could synergize with checkpoint blockade anti-PD1 to prevent tumour growth. All these results indicated that pyroptosis induced by GSDMA3 could trigger robust antitumour immunity and GSDMA3 was a novel target for tumour treatment.

In a word, the two papers recently both published in Nature ${ }^{1,2}$ gave us novel insights that gasdermin $\mathrm{N}$ domains could enrich on the cell membrane to form pores and induce pyroptosis. Antitumour immunity was triggered by inflammation induced by pyroptosis, which was essential for tumour suppressor of gasdermin. Thus, it is a promising way to develop therapeutic strategies depending on gasdermin function, such as the use of the DNA methylation inhibitor decitabine, or combined therapeutics of gasdermin and checkpoint blockade, in order to eradicate tumours by eventually activating robust antitumour immunity.

\section{ACKNOWLEDGEMENTS}

This work was supported by grants from the National Natural Science Foundation of China (No. 81770176) and the Fundamental Research Funds of Zhejiang Sci-Tech University (No. 2019Y001)

\section{REFERENCES}

1. Zhang, Z. et al. Gasdermin E suppresses tumour growth by activating anti-tumour immunity.Nature 579, 415-420 (2020).

2. Wang, Q. et al. A bioorthogonal system reveals antitumour immune function of pyroptosis.Nature 579, 421-426 (2020).

3. Shi, J., Gao, W. \& Shao, F. Pyroptosis: gasdermin-mediated programmed necrotic cell death. Trends Biochem. Sci. 42, 245-254 (2017).

4. Ding, J. et al. Pore-forming activity and structural autoinhibition of the gasdermin family.Nature 535, 111-116 (2016).

5. Xia, X. et al. The role of pyroptosis in cancer: pro-cancer or pro-"host"? Cell Death Dis. 10, 650-662 (2019)

Open Access This article is licensed under a Creative Commons Attribution 4.0 International License, which permits use, sharing, adaptation, distribution and reproduction in any medium or format, as long as you give appropriate credit to the original author(s) and the source, provide a link to the Creative Commons license, and indicate if changes were made. The images or other third party material in this article are included in the article's Creative Commons license, unless indicated otherwise in a credit line to the material. If material is not included in the article's Creative Commons license and your intended use is not permitted by statutory regulation or exceeds the permitted use, you will need to obtain permission directly from the copyright holder. To view a copy of this license, visit http://creativecommons. org/licenses/by/4.0/.

(c) The Author(s) 2020 